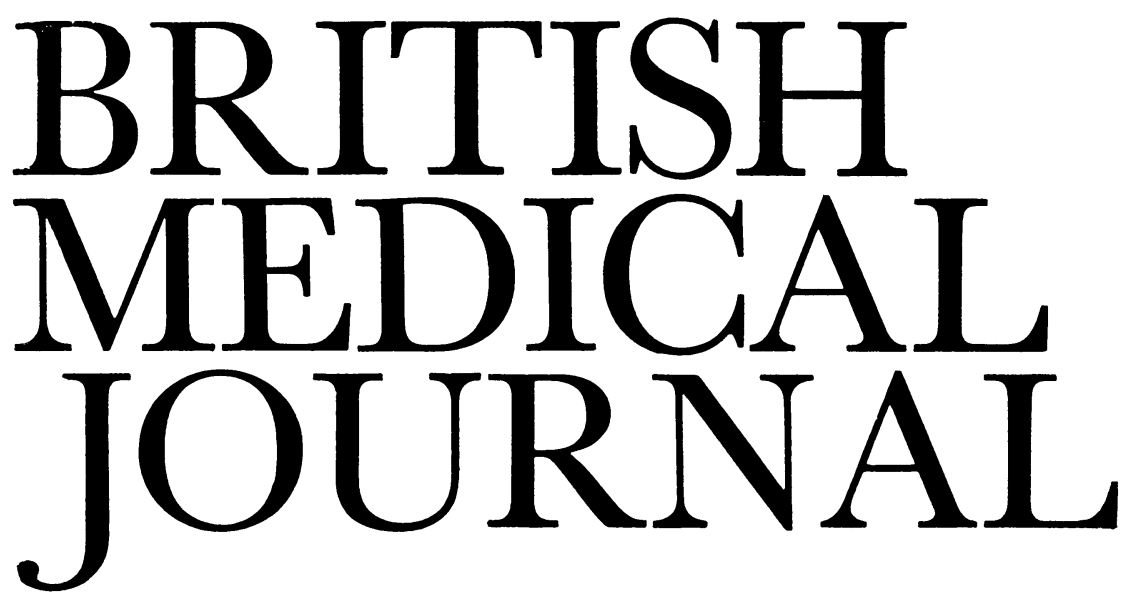

LONDON, SATURDAY 4 FEBRUARY 1984

\title{
Antibiotic guidelines
}

What went wrong with antibiotics? In the heady days of the 1940s and 1950s only a few relatively inexpensive drugs were available but they worked miracle cures and transformed the practice of medicine. Now in the 1980s numerous antibiotics are available, many at considerable cost, including compounds active against fungi and viruses, and yet patients still die of infections which cannot be controlled. Not all the blame for this can be placed on the use or misuse of antibiotics. Advances in medicine and surgery together with social factors have led to a diminution of those infections that are easy to treat and an increasing vulnerable population-vulnerable to infections by organisms of low intrinsic virulence and also by those with natural resistance to many antibiotic substances. ${ }^{1}$ Nevertheless, undoubtedly antibiotic use has contributed to the current state of affairs and led to the emergence of acquired resistance in the important pathogens of man. Many reports from the World Health Organisation and elsewhere have documented the relation of antibiotic use to resistance. ${ }^{2-4}$

The glut of new antibiotics in the past 15 years has given us many effective and valuable compounds, but the extent and complexity of antibiotic development have been such that clinicians have had difficulty in selecting the most appropriate agents for their particular problems. Several attempts have been made to implement a more rational use of antibiotics. ${ }^{5-7}$ In Britain many hospitals produce their own guide books through the antibiotic wonderland, and in others attempts are made to produce an "antibiotic policy" to control the unrestricted use of antibiotics and the devastating effects that this may have on the local microbial flora. The policies have not been entirely successful because clinicians are not too keen on any limitation of their freedom to prescribe. Simplistic policies are unable to cope with the very different problems faced by, say, paediatricians compared with vascular surgeons, or nephrologists responsible for dialysis compared with haematologists treating leukaemia. Restricted reporting of susceptibility tests has an appreciable influence on antibiotic use. ${ }^{8}$ There are also clinical problems of implementing recommendations and the difficulties of educating postgraduates. ${ }^{9}$ Policies have now developed into discussions between clinicians and antibiotic "experts" (those struggling to keep up with the tide of new information on antibiotics). Softening the word "policies" to "guidelines" has also made any guidance more palatable to clinicians.

One effect of the recent cost cutting exercises in National
Health Service hospitals has been that committees are looking hard at antibiotic bills, which have risen continually over the past decade. If an attempt to reduce these is now to be made, the opportunity might be taken to introduce forms of treatment which are more effective for the patient and less destructive to the microbial flora at the same time as saving money. Some practices, such as the greatly increased use of prophylactic regimens in different forms of surgery and the blind treatment of patients with neutropenia and of those undergoing peritoneal dialysis, need particular scrutiny. Similarly, intensive therapy units, special care baby units, and haematological units are departments where updating and re-evaluation of current practices might be done. Doctors tend to assume that the latest antibiotic on the market has added benefits over more established agents yet retains all the virtues of the latter. This is not necessarily the case: raising the activity of an antibiotic against one group of organisms usually leads to loss of activity against others. The usefulness of the older antibiotics such as erythromycin and vancomycin, which in recent years have been put on the shelf, needs to be examined in the context of present problems.

Developing rational and inexpensive guidelines will entail detailed discussions among various groups of clinicians, pharmacists, medical microbiologists, and the dwindling numbers of infectious disease physicians. In Britain medical microbiologists form the largest body of medical opinion able to give informed opinion on current antibiotics, opinions often derived from personal experience in evaluating the compound and adopting it for susceptibility testing in the laboratory. They will need to spend considerable time and effort to ensure that the most clinically useful guidelines are adopted as well as in reducing the expense. Most of these discussions will have to be held locally, and Kunin has produced some useful detailed instructions on how to prepare an audit of antibiotic use as well as guidelines. ${ }^{1011}$

National guidelines have been laid down centrally in several eastern European and developing countries. ${ }^{12}$ Nevertheless, very few national guidelines are available in Britain which are derived from a consensus of opinion. A notable exception is the report on prevention of endocarditis arising from dental procedures, in which a group of cardiologists, dentists, and medical microbiologists produced a reasonable, practical, and effective set of suggestions. ${ }^{13}$ Many other areas of practice are suitable for national recommendations, but 
meanwhile there are as many men as opinions about what constitutes rational antibiotic treatment. Thus guidelines will be mainly derived from personal predilections and preferences.

\section{J D Williams}

Professor of Medical Microbiology,

London Hospital Medical College,

London $\mathrm{E} 12 \mathrm{AD}$

${ }^{1}$ Geddes AM. The impact on clinical practice of antibiotic-resistant micro-organisms. In: Stuart-Harris Sir CH, Harris DM, eds. The control of antibiotic-resistant bacteria. London: Academic Press, 1982: $1-16$.

${ }^{2}$ Mouton RP, Glerum JH, van Loenen AC. Relationship between antibiotic consumption and frequency of antibiotic resistance of four pathogens-a seven-year survey. F Antimicrob Chemother 1976;2:9-19.

3 Ayliffe GAJ. Use of antibiotics and resistance. In: Geddes AM, Williams JD, eds. Current antibiotic therapy. London: Churchill Livingstone, 1973:53-63.

- World Health Organisation. Surveillance for the prevention and control of health hazards due to antibiotic resistant enterobacteria. Geneva: World Health Organisation, 1978. (WHO Technical Report Series, No 624.)

${ }^{5}$ Anonymous. Good antimicrobial prescribing. A Lancet review. London: The Lancet, 1982.

${ }^{6}$ Buckwold FJ, Ronald AR. Antimicrobial misuse-effects and suggestions for control. F Antimicrob Chemother 1979;5:129-36.

${ }^{7}$ Cooke D, Salter AJ, Phillips I. Antimicrobial misuse, antibiotic policies and information resources. F Antimicrob Chemother 1980;6:435-43.

${ }^{8}$ Casewell MW, Pugh S, Dalton MT. Correlation of antibiotic usage with an antibiotic policy in a urological ward. Fournal of Hospital Infection $1981 ; 2: 55-61$.

${ }^{8}$ Harvey K, Stewart R, Hemming M, Moulds R. Use of antibiotic agents in a large teaching hospital. The impact of antibiotic guidelines. Med f A ust 1983;ii :217-21.

10 Kunin CM. Evaluation of antibiotic usage; a comprehensive look at alternative approaches. In: Ninet L, Bost PE, Bouanchaud $\mathrm{DH}$, Florent J, eds. The future of antibiotherapy and antibiotic research. London: Academic Press, 1981:127-39.

${ }^{11}$ Kunin CM. Problems of antibiotic usage. Definitions, causes, and proposed solutions. Ann Intern Med 1978;89:802-5.

${ }^{12}$ Modr Z. Statutory control of antibiotic use in man versus voluntary restriction. In: Stuart-Harris Sir CH, Harris DM, eds. The control of antibiotic-resistant bacteria. London: Academic Press, 1982:211-26.

13 Working Party of the British Society for Antimicrobial Chemotherapy. The prophylaxis of infective endocarditis. Lancet 1982;ii:1323-6.

\section{Supraventricular tachycardias}

The term "supraventricular tachycardia" includes all those arrhythmias arising from the atrial myocardium and atrioventricular junctional tissue. Arrhythmias such as atrial fibrillation, atrial flutter, and atrial tachycardia are due to electrical instability of the atrial myocardium, are most often associated with organic heart disease, and are more commonly seen in older patients. In contrast, tachycardias occurring as a result of abnormalities of atrioventricular conduction, either functional (for example, multiple atrioventricular nodal pathways) or anatomical (for example, accessory bundles), are usually seen in patients without organic heart disease and occur at all ages.

Over the past decade intracardiac studies have shown many mechanisms for these tachycardias, most of which are caused by continuous circulation of depolarising activity and are known as "re-entrant." A characteristic of re-entrant tachycardias is that they may be started or stopped by electrical stimulation; this correlates with the clinical observation of sudden onset and cessation in most patients. ${ }^{2}$ In a few tachycardia may be incessant. To some extent the mechanism of the tachycardia may determine the frequency and duration of attacks.

We still know little about the clinical course of the different types of arrhythmia, but what evidence there is suggests that in individual patients the frequency and severity of untreated episodes of tachycardia remain unchanged over many years. ${ }^{3}$ Many patients with infrequent or brief episodes do not need treatment. A substantial proportion, however, are troubled by recurrent attacks and require long term treatment. This most often takes the form of oral antiarrhythmic drugs to suppress events which may trigger tachycardia (for example, extrasystoles), to control the ventricular response to continuing tachycardia (as in atrial fibrillation), or to modify the circuit so that it can no longer sustain tachycardia despite the continued occurrence of triggering events. Though many patients may be treated successfully with conventional drugs, alternatives must be considered for the minority who are not controlled. Of the more recently available antiarrhythmic drugs, amiodarone is perhaps the most widely used. This drug is of great value in patients with tachycardias which fail to respond to other drugs, ${ }^{45}$ but a high incidence of side effects limits its value for long term treatment. ${ }^{6}{ }^{7}$ If treatment with amiodarone is unsuccessful newer investigational drugs should be considered. One such group, with cellular properties similar to those of quinidine and disopyramide ${ }^{8}$ but with distinctly different clinical effects, is of particular interest. Flecainide is the most widely known of these drugs in Britain and offers considerable promise in the termination and prevention of supraventricular tachycardias of all types. ${ }^{9}$

In selected patients with recurrent paroxysmal tachycardias the choice of an effective preventive drug may be helped by intracardiac stimulation studies-an approach already accepted as an essential part of the management of ventricular tachycardia. ${ }^{10}$ It allows the antiarrhythmic effect of several drugs to be tested individually over a short period of time, thus avoiding the laborious and often ineffective process of empirical drug trials. Suppression of a tachycardia induced electrically in the laboratory predicts a favourable long term response to oral treatment. ${ }^{11} 12$

If antiarrhythmic drugs fail, are not appropriate (for example, in pregnancy), or are not tolerated several approaches may be considered. Surgical ablation of the bundle of His or accessory bundle will prevent rapid ventricular rates in response to atrial tachycardias or interrupt a re-entrant pathway. These procedures have been discussed in a previous leading article. ${ }^{13}$ More recently, several groups have successfully excised the focus of paroxysmal atrial tachycardia. ${ }^{14} 15$

Surgery is usually considered as a last resort. Less drastic is the use of pacemakers, which can suppress tachycardia or terminate recurrent paroxysms. The former application is rarely successful in the long term, but tachycardia termination pacemakers have proved themselves valuable for patients with recurrent tachycardias. ${ }^{16}$ External versions of such pacemakers have been used for several years, and recently a fully implantable and automatic version has become available. ${ }^{1718}$ These pacemakers "recognise" tachycardia as a fast rate or, in the more refined devices, as a sudden change to a rapid rate. They respond by rapid overdrive or premature stimulation designed to interrupt tachycardia. At present the termination algorithms are relatively crude and often allow the tachycardia to continue for some time before it is stopped. Physiological and technical problems are, however, likely to be overcome very shortly. Improvements in design and growing evidence of both the safety and efficacy of these devices seem likely to lead to their more liberal use as their cost and complexities are balanced against the side effects inevitable with drug treatments.

Treatment with pacemakers is not, however, appropriate in patients with frequently repetitive or continuous tachycardias -and some tachycardias (such as atrial fibrillation and some 